\title{
The rotating cement kiln 3D computer model oriented toward solving thermal nondestructive testing problems
}

\author{
by V. G. Torgunakov, M. S. Sukhanov, V. P. Vavilov \\ and N. M. Yamanaev
}

Tomsk Polytechnic University, Russia, 634028, Tomsk, 28, Savinykh St., 3

\begin{abstract}
The three-dimensional mathematical model of heat transfer in a rotating cement kiln is proposed. Both heat conduction and mass transfer phenomena are taken into account. it is assumed that, due to good mechanical mixing, clinker temperature is constant along both radial and azimuthal coordinates.

The numerical model is realised as a computer program that is used in solving an appropriate inverse heat transfer problem when inspecting insulation material loss in industrial cement kilns.
\end{abstract}

\section{Introduction}

In infrared thermographic nondestructive testing (TNDT) of cement kilns, surface temperature is monitored with an infrared device to supply information about possible kiln jacket defects. Mathematically, this requires solving of an inverse heat transfer problem where experimental temperature distributions serve as input data in retrieving jacket material loss [1]. As the first step in solving such inverse problem, we have developed the direct computer model of heat transfer in a cement kiln [2] that allows computing temperatures both on kiln surface and inside a kiln jacket. The model involves numerical calculation of temperature within a jacket multilayer annular structure that contains a dry substance called clinker to be roasted in a kiln (figure1). This study is devoted to improving the previous model.

The following physical processes are taken into account when performing computations:

- convective heat exchange with the ambient that occurs on a kiln external surface;

- heat exchange by heat conduction inside a kiln jacket material;

- convective heat exchange with the gas that flows inside a kiln jacket;

- axial rotation of a kiln;

- clinker material mass transfer.

\section{Theory and modelling}

The whole entity is presented as a population of Dirichlet cells in three-dimensional cylindrical co-ordinates ( $r-\varphi$-z-geometry). The heat balance equations are recorded for each cell to form a finite-differential model of heat transfer in a cement kiln. The known numerical split-method is used to solve these equations.

The serious mathematical problem not yet solved is an appropriate choice of temporal steps needed to analyse both heat conduction and mass transfer phenomena while a kiln is rotating. To compute mass transfer in a clinker, a less than one second temporal step is needed to match kiln rotation. On the other hand, reaching a stationary regime for a typical industrial kiln would require following a process up to hundred hours. As a result, too long computations could be involved even if using a rather powerful computer.

However, the results obtained by using a three dimensional model have shown that, in a typical cement kiln, defects that appear on the kiln internal surface will influence the external surface temperature distribution only $8.3 \mathrm{hrs}$ after a heat transfer process starts that corresponds to about 500 revolutions of a kiln. Therefore, for correct computation of external temperature, it could be assumed that a clinker mass is well mixed and clinker temperature varies only by the Z-coordinate. This assumption simplifies significantly timing of a problem 
when calculating mass transfer phenomena without worsening temperature calculation accuracy on the external surface. One of $(\mathrm{i}, \mathrm{j}, \mathrm{k})$ Dirichlet cells is shown in figure 2 for a single spatial node.

Below, there are equations that describe heat transfer in a cell along the radius $\mathrm{R}$.

For the Dirichlet cells that do not contact with a gas ambient the heat balance equation is written in the following form:

$$
\begin{gathered}
\left(C \rho_{i-\frac{1}{2}, j, k} V_{i-\frac{1}{2}, j, k}+C \rho_{i+\frac{1}{2}, j, k} V_{i+\frac{1}{2}, j, k}\right) \frac{U_{i, j, k}^{n+1}-U_{i, j, k}^{n}}{\Delta t}= \\
=\lambda_{i-\frac{1}{2}, j, k} \frac{U_{i-1, j, k}^{n+1}-U_{l, j, k}^{n+1}}{l_{i}} S_{i-\frac{1}{2}}+\lambda_{i+\frac{1}{2}, j, k} \frac{U_{i+1, j, k}^{n+1}-U_{i, j, k}^{n+1}}{l_{i+1}} S_{i+\frac{1}{2}}
\end{gathered}
$$

where $C \rho_{i-\frac{1}{2}, j, k}$ is the product of the density by the heat capacity for the one half of the Dirichlet cell which locates between radii $R_{i}$ and $\frac{R_{i}+R_{i-1}}{2}$ (radial distance between nodes could vary between layers); $C \rho_{i+\frac{1}{2}, j, k}$ is the product of the density by the heat capacity for the one half of the Dirichlet cell which locates between radii $R_{i}$ and $\frac{R_{i}+R_{i+1}}{2}$; $V_{i-\frac{1}{2}, j, k}, V_{i-\frac{1}{2}, j, k}$ are the volumes of the above-mentioned Dirichlet cell halves; $\lambda_{i-\frac{1}{2}}, \lambda_{i+\frac{1}{2}}$ are the heat conduction coefficients for both halves of a cell; $S_{i-\frac{1}{2}}, S_{i+\frac{1}{2}}$ are heat exchange areas that appear between the cell $\mathrm{i}, \mathrm{j}, \mathrm{k}$ and adjacent cells $\mathrm{i}-\mathrm{l}, \mathrm{j}, \mathrm{k}$ and $\mathrm{i}+\mathrm{l}, \mathrm{j}, \mathrm{k}$ respectively; $U_{i, j, k}^{n+1}, U_{i, j, k}^{n}$ are the temperatures in the Dirichlet cells which correspond to the next and the former temporal layers respectively; $l_{i}=R_{i}-R_{i-1}$ is the distance between centers of Dirichlet cells along the radius; $\Delta t$ is the time period between two adjacent temporal layers.

The equation (1) has been derived by using the following assumptions:

- borders between zones having different thermal properties should run only along grid lines;

- numerical grid is uniform along the axes $Z$ and $\varphi$ but could be non-uniform along the axis $\mathrm{R}$.

Within a Dirichlet cell, the border between zones having different thermal properties runs on the grid surfaces which are parallel to the axis of R- $\varphi$ or to a R-Z surface; therefore, the left and the right part of equation (1) are divided into the appropriate number of members.

For the Dirichlet cells which locate on the internal kiln surface the heat exchange equations should be written in the different form:

$$
\begin{gathered}
\left(C \rho_{i-\frac{1}{2}, j, k} V_{i-\frac{1}{2}, j, k}+C \rho_{k}^{k l} V_{k}^{k l}\right) \frac{U_{i, j, k}^{n+1}-U_{i, j, k}^{n}}{\Delta t}= \\
=\lambda_{l-\frac{1}{2}, j, k} \frac{U_{i-1, j, k}^{n+1}-U_{i, j, k}^{n+1}}{l_{i-1}^{n+1}} S_{i-\frac{1}{2}}+ \\
+\lambda_{k}^{k l} \frac{U_{k}^{k l, n+1}-U_{i, j, k}^{n+1}}{l_{i-1}} S_{i+\frac{1}{2}} \theta+\alpha_{1}\left(U_{G}(Z)-U_{i, j, k}^{n+1}\right) S_{i}(1-\theta)
\end{gathered}
$$


where $C \rho_{k}^{k l}$ is the product of the density by the heat capacity for the clinker cell adjacent to the particular Dirichlet cell; $V_{k}^{k l}$ is the clinker cell volume; $U_{k}^{k l, n+1}$ is the clinker cell temperature on the $n+1$-th temporal layer; $\theta$ is the part of the time during which Dirichlet cells are under the clinker; $\alpha_{1}$ is the effective heat exchange coefficient that takes into account convective and radial heat exchange with the gas flowing in a kiln; $U_{G}(Z)$ is the gas temperature as a function of the $Z$ variable.

To describe heat transfer along the Z-axis within a clinker, one could introduce the following equation:

$$
\begin{gathered}
C \rho_{k}^{k l} V_{k}^{k l} \frac{U_{k}^{k l, n+1}-U_{k}^{k l, n}}{\Delta t}=\lambda_{k}^{k l} \frac{U_{k-1}^{k l, n+1}-U_{k}^{k l, n+1}}{l_{k}} S_{k}+ \\
+\lambda_{k}^{k l} \frac{U_{k+1}^{k l, n+1}-U_{k}^{k l, n+1}}{l_{k}} S_{k}+C_{\omega} \varpi\left(U_{k-1}^{k l, n+1}-U_{k}^{k l, n+1}\right) \rho_{k}^{k l},
\end{gathered}
$$

where $C_{w} w$ is the kiln output in $\mathrm{kg}$ per second.

\section{Discussion and conclusion}

The mathematical model described with equations (1-3) is more sophisticated than our previous model [2] due to more correct organisation of a run process along the radius. Computer realisation of this model has demonstrated that one could use greater temporal steps without loosing accuracy. Thus, a computation rate could be enhanced by two or three orders.

The theoretical results discussed above are used in Tomsk Polytechnic University in developing the intellectual TNDT system orientated toward inspection of industrial cement kilns. The typical test situation appearing on the operator's monitor is presented in figure 3 (also in color). The program window demonstrates the fragment of the recorded temperature distribution on the kiln external surface along with the kiln insulation cross-section where insulation material loss is determined by processing experimental data and using theoretical results for material loss calibration. The temperature profile, as well as extreme temperature values, is also supplied to make easier evaluation of kiln operation conditions by an operator.

\section{REFERENCES}

[1] SOLOVYEV (A. V.) and SOLOVYEVA (E. V.) - The Dirichlet Cell Method to Solve Gas Dynamic Equations in the Cylindrical Coordinates. Preprint of Institute of Applied Mathematics \#80 U.S.S.R., Moscow, 1986.- 32 p. (in Russian).

[2] SUKHANOV (M. S.) and TORGUNAKOV (V. G.) - The Computer Model of Thermal Processes in a Cement Kiln. In: Proc. 7-th European Conf. on Non-Destructive Testing, Denmark, 1998, p.347. 


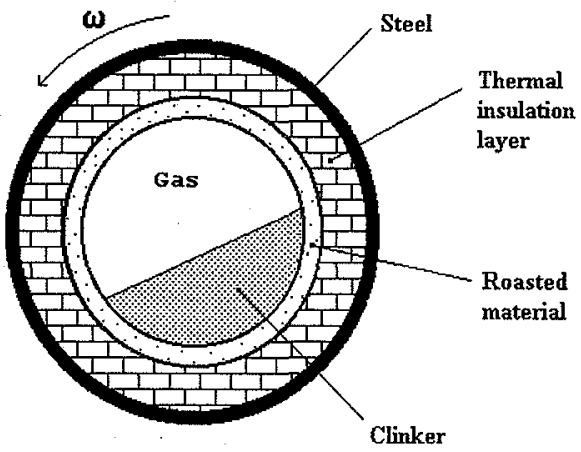

Fig. 1. Cross section of a cement kiln

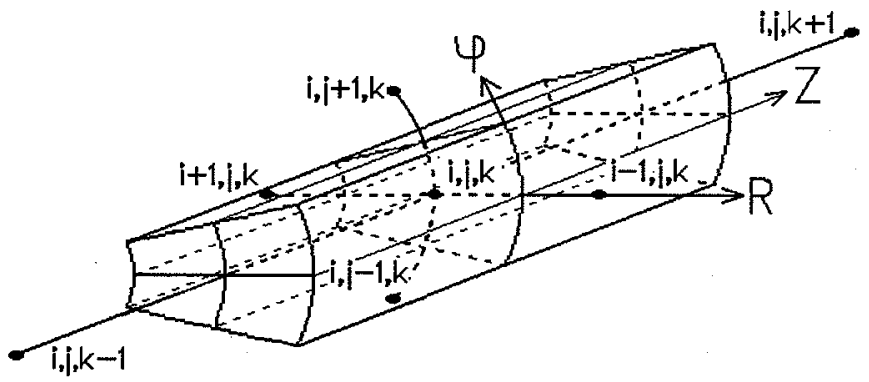

Fig. 2. Dirichlet cell for the $\mathbf{i}, \mathbf{j}, \mathbf{k}$ grid node

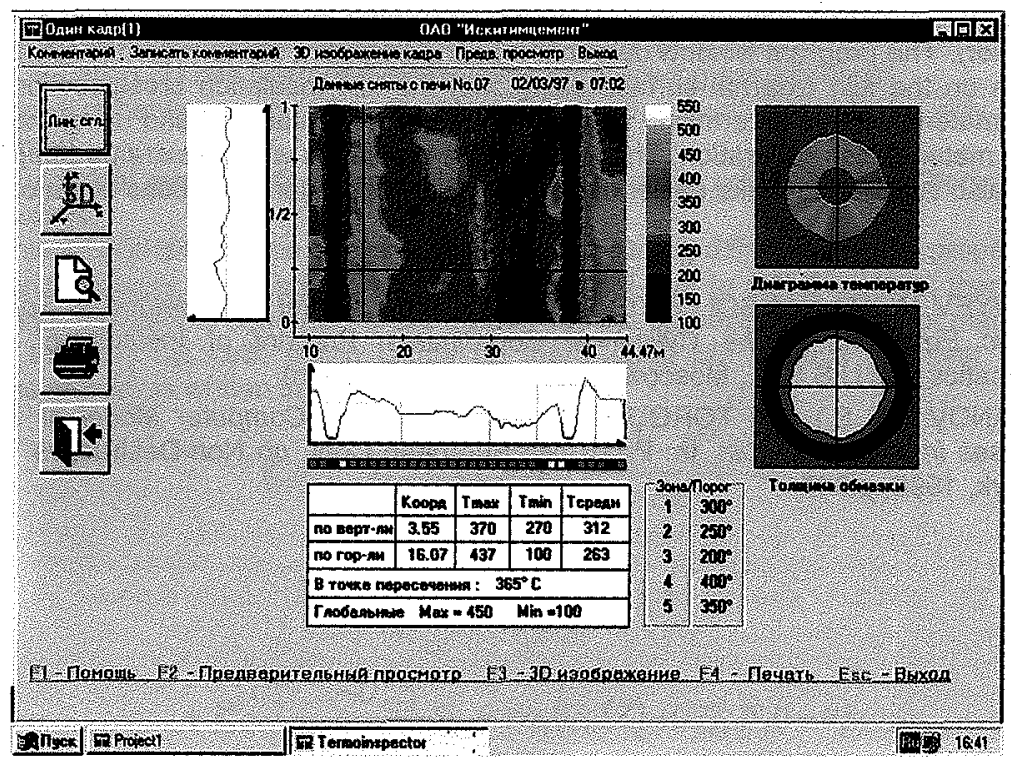

Fig. 3. Example of experimental data presentation in the inspection of a cement kiln 